\title{
Reavaliação da suscetibilidade de Candida à anfotericina B: estudo comparativo com isolados de três hospitais do Estado do Rio Grande do Sul
}

\author{
Reevaluation of Candida susceptibility to amphotericin B: \\ comparative study using isolates from three hospitals \\ in the State of Rio Grande do Sul
}

\author{
Everton Boff ${ }^{1}$, Paulo Guilherme Markus Lopes ${ }^{1}$, Tatiana Spader ${ }^{1}$, Liliane Alves Scheid ${ }^{1}$, \\ Érico Loreto", Nara Frasson Dal Forno ${ }^{2}$, Valério Aquino ${ }^{3}$, Luiz Carlos Severo ${ }^{4}$, \\ Janio Morais Santurio ${ }^{5}$ e Sydney Hartz Alves ${ }^{5}$
}

\begin{abstract}
RESUMO
Comparou-se a suscetibilidade à anfotericina B de Candida spp isoladas de candidemias, sendo: 41 do Hospital Universitário de Santa Maria, 56 do Hospital de Clínicas de Porto Alegre e 47 da Santa Casa, Complexo Hospitalar de Porto Alegre. Os testes foram baseados no documento M27-A2 do Clinical Laboratory Standards Institute. Todavia, foram empregadas 20 concentrações de anfotericina B, variáveis entre 0,1 e 2 $\mu \mathrm{g} / \mathrm{ml}$. Os testes foram realizados nos meios RPMI 1640 com glicose, antibiotic medium 3 e yeast nitrogen base dextrosado. 0 caldo antibiotic medium 3 gerou amplas faixas de concentrações inibitórias mínimas e concentrações fungicidas mínimas quando comparado aos demais. As variações de suscetibilidade entre os hospitais foram melhor detectadas no antibiotic médium 3; os isolados do Hospital Universitário de Santa Maria evidenciaram menor sensibilidade do que os da Santa Casa, Complexo Hospitalar de Porto Alegre $(\mathrm{p}<0,05)$. As causas das variações de suscetibilidade não foram avaliadas mas apontam para a necessidade de vigilância da suscetibilidade a anfotericina $\mathrm{B}$.
\end{abstract}

Palavras-chaves: Suscetibilidade. Anfotericina B. Candida. Candidemia.

\begin{abstract}
Susceptibility to amphotericin B was compared between isolates of Candida spp that were obtained from candidemia cases as follows: 41 from Hospital Universitário de Santa Maria, 56 from Hospital de Clínicas, Porto Alegre, and 47 from the Santa Casa hospital complex, Porto Alegre. The tests were based on the document M27-A2 from the Clinical Laboratory Standards Institute, but with 20 concentrations of amphotericin B ranging from 0.1 to $2 \mu \mathrm{g} / \mathrm{ml}$. The tests were carried out using RPMI 1640 medium with glucose, antibiotic medium 3 and yeast nitrogen base-dextrose. The antibiotic medium 3 broth generated wide ranges of minimum inhibitory concentrations and minimum fungicidal concentrations in relation to the other agents. The variations in susceptibility between the hospitals were best detected in antibiotic medium 3. The isolates from Hospital Universitário de Santa Maria showed lower susceptibility than did those from the Santa Casa hospital complex, Porto Alegre $(\mathrm{p}<0.05)$. The causes of the susceptibility variations were not assessed but they indicate the need for surveillance regarding the susceptibility to amphotericin $B$.
\end{abstract}

Key-words: Susceptibility. Amphotericin B. Candida. Candidemia.

Entre as infecções micóticas sistêmicas, as fungemias têm evidenciado incidência crescente; entre 1979-2000 registrouse, nos Estados Unidos, um aumento de 207\%; as fungemias causadas por Candida spp ocupam o quarto lugar entre todas as infecções do tecido sanguíneo ${ }^{25}$. Nas candidemias as taxas de mortalidade bruta são de $57 \%$ e de mortalidade atribuída atingem 38\% e de modo geral, o tempo de hospitalização aumenta em média 48 dias $^{31}$. Candida spp é o único microrganismo

\footnotetext{
1. Curso de Pós Graduação em Ciências Farmacêuticas, Universidade Federal de Santa Maria, Santa Maria, RS. 2. Laboratório de Microbiologia, Hospital Universitário de Santa Maria, Santa Maria, RS. 3. Laboratório de Análises Clínicas, Hospital de Clínicas de Porto Alegre, Porto Alegre, RS. 4. Departamento de Medicina Interna, Faculdade de Medicina, Universidade Federal do Rio Grande do Sul, Porto Alegre, RS. 5. Departamento de Microbiologia e Parasitologia, Universidade Federal de Santa Maria. Santa Maria, RS. Endereço para correspondência: Dr. Sydney Hartz Alves. Rua dos Andradas 1985/201, 97010-033 Santa Maria, RS.

Telefax: 5555 3220-8906

e-mail: hartzsa@smail.ufsm.br

Recebido para publicação em: 01/06/2007

Aceito em: 17/12/2007
} 
que, independentemente, influencia no desfecho das infecções sanguíneas estando, por isto, associado a taxas de mortalidade mais elevadas do que as observadas com outros patógenos ${ }^{2326}$.

As principais causas das falhas da terapêutica antifúngica sistêmica incluem: a) doses inadequadas; b) absorção, distribuição ou metabolismo deficientes; c) interações medicamentosas; d) neutropenia grave; e) presença de corpo estranho; f) emergência do fenômeno da resistência aos antifúngicos ${ }^{3}$.

A capacidade dos testes de suscetibilidade em detectar fungos leveduriformes do gênero Candida resistentes a antifúngicos azólicos, está bem documentada ${ }^{51011523}$; por outro lado, a avaliação da suscetibilidade de Candida spp à anfotericina B tem sido mais dificultada devido a ausência de pontos de corte (breakpoints) indicadores de resistência ${ }^{10131522}$.

A anfotericina B é um antifúngico poliênico, disponível como fármaco de uso sistêmico desde $1956 \mathrm{e}$, apesar de sua conhecida toxicidade, é ainda largamente utilizada na terapêutica das micoses sistêmicas ${ }^{23591021}$.

Este estudo foi proposto para comparar as variações da suscetibilidade de Candida spp, isoladas de episódios de candidemia, frente à anfotericina $\mathrm{B}$, em três hospitais públicos do Rio Grande do Sul. Considerando que o caldo RPMI (Roswell Park Memorial Institute) 1640 indicado na técnica padronizada, compacta a faixa de variação das concentrações inibitórias mínimas (CIMs) ${ }^{2425}$ no presente trabalho empregamos também 0 caldo antibiotic medium $3(\mathrm{AM} 3)^{17} \mathrm{e} 0$ caldo yeast nitrogen base dextrosado $(\mathrm{YNBd})^{16}$. Objetivando-se estabelecer rigorosa comparação entre os resultados, os ensaios foram realizados com concentrações de anfotericina B variáveis entre si em $0,1 \mu \mathrm{g} / \mathrm{ml}$ e não apenas com concentrações progressivas ao dobro, como preconiza a metodologia padronizada.

\section{MATERIAL E MÉTODOS}

Instituições envolvidas. Os microrganismos incluídos neste estudo foram isolados no Hospital Universitário de Santa Maria (Hospital A), Hospital de Clínicas de Porto Alegre (Hospital B) e Santa Casa - Complexo Hospitalar de Porto Alegre (Hospital C).

Microrganismos. Cento e quarenta e quatro isolados de Candida spp foram incluídos. Nos três centros incluídos no estudo, a identificação dos isolados de Candida utilizou o sistema ID-32C (Biomérieux). De acordo com os hospitais, as espécies de Candida incluíram: Hospital A $(\mathrm{n}=41)$, Candida albicans (29), Candida tropicalis (8), Candida krusei (2), Candida parapsilosis (1), Candida glabrata (1); Hospital B ( $n=56)$, Candida albicans (23), Candida parapsilosis (20), Candida tropicalis (11), Candida glabrata (1), Candida guilliermondii (1); Hospital C ( $\mathrm{n}=47)$, Candida albicans (18), Candida parapsilosis (18), Candida tropicalis (4), Candida pelliculosa (3), Candida glabrata (2), Candida dubliniensis (1), Candida guilliermondii (1).

Testes de suscetibilidade. Foram realizados pela técnica de microdiluição em caldo, de acordo com o protocolo M27$\mathrm{A} 2{ }^{17}$. Todos os isolados foram testados frente à anfotericina $\mathrm{B}$
(Sigma) em três meios de cultura: a) caldo RPMI 1640 (com L-glutamina e sem bicarbonato) (Gibco) suplementado com glicose $(20 \mathrm{~g} / \mathrm{L}$ ) e tamponado com MOPS [ácido 3-(N-morfolino propano sulfônico)] (Sigma) até concentração final de $165 \mathrm{mM}$; b) antiobiotic medium 3 (AM3) ${ }^{17}$ (BBL) suplementado com glicose $2 \%$ e tamponado $(\mathrm{pH}=7)$ com $\mathrm{K}_{2} \mathrm{HPO}_{4}$ e $\mathrm{KH}_{2} \mathrm{PO}_{4}$; c) yeast nitrogen base ${ }^{16}$ (Difco) preparado de acordo com as instruções do fabricante; após reconstituição foi suplementado com glicose $0,5 \%$ e tamponado com MOPS ( $\mathrm{pH}=7$ ) $165 \mathrm{mM}$. Os 3 meios foram esterilizados por filtração em membranas de $0,22 \mu \mathrm{m}$ (millipore). As soluções estoque de anfotericina B foram preparadas solubilizando-se 0 antifúngico na forma de pó em dimetilsulfóxido e conservando-as em freezer a $-70^{\circ} \mathrm{C}$ até o momento do uso. As diluições do antifúngico foram realizadas em cada um dos meios utilizados, onde o número de concentrações foi ampliado; adotou-se uma escala unidecimal cujas concentrações variaram de $0,1 \mu \mathrm{g} / \mathrm{mL}$ a $2 \mu \mathrm{g} / \mathrm{mL}$. A concentração de $4 \mu \mathrm{g} / \mathrm{mL}$ também foi avaliada mas, neste intervalo, dispensou-se a inclusão de concentrações intermediárias. Os testes foram realizados em placas de microdiluição, nas quais, alíquotas de $0,1 \mathrm{~mL}$ de anfotericina B 2X concentrada eram dispensadas. As microplacas eram fechadas, envoltas em parafilme e, congeladas a $-70^{\circ} \mathrm{C}$, até o uso. Os inóculos eram preparados a partir de cultivos de 24-48h em ágar Sabouraud dextrose, suspendendo-se as células fúngicas em água destilada estéril, cujas turvações eram ajustadas e padronizadas espectrofotometricamente conforme preconiza o documento M27-A2; finalmente, eram diluídas no mesmo meio onde seriam testadas. No dia dos testes, placas de microdiluição contendo 100 $\mu \mathrm{L}$ das concentrações de anfotericina B eram inoculadas com $100 \mu \mathrm{L}$ do inóculo diluído no mesmo meio, resultando em $0.5 \times 10^{3}$ a $2,5 \times 10^{3}$ células $/ \mathrm{mL}$ em cada escavação. Os testes eram realizados em triplicata e, a seguir, as microplacas eram incubadas a $35^{\circ} \mathrm{C}$. Após $48 \mathrm{~h}$ de incubação, determinava-se as concentrações inibitórias mínimas (CIMs); estas foram definidas como a menor concentração de anfotericina B que determinasse $100 \%$ de inibição do crescimento. Candida parapsilosis ATCC 22019 e Candida krusei ATCC 6258 foram incluídas como controle de qualidade dos ensaios; controles de esterilidade do meio e do meio com anfotericna B, foram também realizados. As concentrações fungicidas mínimas (CFMs) eram obtidas pela transferência de $0,1 \mathrm{~mL}$ de cada concentração que inibiu o crescimento fúngico, para a superfície de ágar Sabouraud dextrose. Após incubação de 48-72h, a CFM foi definida como a menor concentração de anfotericina B capaz de causar a morte de $100 \%$ das células ${ }^{26}$.

Análise estatística. Nas comparações envolvendo duas variáveis, empregou-se o teste não paramétrico de Wilcoxon; quando as comparações envolviam três ou mais variáveis, utilizouse o teste não paramétrico de Kruskal-Wallis.

\section{RESULTADOS}

As concentrações inibitórias mínimas e concentrações fungicidas mínimas dos 144 isolados de Candida spp procedentes de episódios de candidemia, de três hospitais do Rio Grande do Sul, 
encontram-se dispostas nas Tabelas 1, 2 e 3, conforme o meio de cultura empregado.

Neste estudo, o maior número de concentrações avaliadas, resultou em amplas faixas de variação das CIMs; entretanto, variações inerentes aos meios de cultura foram detectadas: no RPMI 1640 observou-se uma faixa de CIMs com oito concentrações; o caldo YNBd forneceu uma faixa com nove concentrações e, no AM3, a faixa de CIMs envolveu 11 concentrações de anfotericina B (Tabelas 1, 2 e 3).

Em relação as faixas de variação das CFMs, verificou-se que estas foram, com os três meios, mais amplas do que as obtidas para as CIMs. Similarmente ao observado nas CIMs, o caldo RPMI 1640 evidenciou uma faixa de variação de concentração

Tabela 1 - Avaliação da suscetibilidade de Candida sppprocedentes de três hospitais do Rio Grande do Sul, frente à anfotericina $\mathrm{B}$, utilizando-se o Caldo RPMI 1640.

\begin{tabular}{|c|c|c|c|c|c|c|c|}
\hline \multirow[b]{2}{*}{ Espécies } & \multirow[b]{2}{*}{ Hospitais } & \multicolumn{3}{|c|}{ CIM $(\mu \mathrm{g} / \mathrm{ml})$} & \multicolumn{3}{|c|}{$\operatorname{CFM}(\mu \mathrm{g} / \mathrm{ml})$} \\
\hline & & variação & CIM50 & CIM90 & variação & CIM50 & $\overline{\text { CIM90 }}$ \\
\hline Candida & A & $0,1-0,7$ & 0,4 & 0,6 & $0,2-0,8$ & 0,6 & 0,7 \\
\hline \multirow[t]{2}{*}{ albicans } & B & $0,1-0,6$ & 0,5 & 0,6 & $0,1-0,9$ & 0,6 & 0,8 \\
\hline & C & $0,1-0,7$ & 0,4 & 0,6 & $0,5-0,7$ & 0,6 & 0,7 \\
\hline Candida não & A & $0,1-0,6$ & 0,5 & 0,6 & $0,1-0,9$ & 0,7 & 0,9 \\
\hline \multirow[t]{2}{*}{ albicans } & B & $0,1-0,8$ & 0,4 & 0,7 & $0,2-1,0$ & 0,7 & 1,0 \\
\hline & $\mathrm{C}$ & $0,1-0,6$ & 0,3 & 0,5 & $0,2-0,6$ & 0,5 &,- 6 \\
\hline \multirow[t]{3}{*}{ Total } & A & $0,1-0,7$ & 0,5 & 0,6 & $0,1-0,9$ & 0,7 & $\overline{0,9}$ \\
\hline & B & $0,1-0,8$ & 0,5 & 0,7 & $0,1-1,0$ & 0,7 & 1,0 \\
\hline & $\mathrm{C}$ & $0,1-0,7$ & 0,4 & 0,6 & $0,2-0,7$ & 0,6 & 0,6 \\
\hline \multicolumn{8}{|c|}{ Candida parapsilosis } \\
\hline ATCC 22019 & - & $0,3-0,4$ & ND & ND & $0,3-0,5$ & ND & ND \\
\hline \multicolumn{8}{|c|}{ Candida krusei } \\
\hline ATCC 6258 & - & 0,6 & ND & ND & 0,8 & ND & ND \\
\hline
\end{tabular}

CIM: concentração inibitória mínima, CFM: concentração fungicida mínima, ND: não determinado. A (Hospital Universitário de Santa Maria), B (Hospital de Clínicas de Porto Alegre), C (Santa Casa, Complexo Hospitalar de Porto Alegre).

Tabela 2 - Avaliação da suscetibilidade de Candida sppprocedentes de três hospitais do Rio Grande do Sul, frente à anfotericina $\mathrm{B}$, utilizando-se o Caldo AM3.

\begin{tabular}{|c|c|c|c|c|c|c|c|}
\hline \multirow[b]{2}{*}{ Espécies } & \multirow[b]{2}{*}{ Hospitais } & \multicolumn{3}{|c|}{$\mathrm{CIM}(\mu \mathrm{g} / \mathrm{ml})$} & \multicolumn{3}{|c|}{$\operatorname{CFM}(\mu \mathrm{g} / \mathrm{ml})$} \\
\hline & & variação & CIM50 & CIM90 & variação & CIM50 & CIM90 \\
\hline \multirow[t]{3}{*}{$\overline{\text { Candida albicans }}$} & $\bar{A}$ & $0,3-0,9$ & 0,6 & 0,9 & $0,4-1,3$ & 0,8 & 1,3 \\
\hline & B & $0,2-0,8$ & 0,6 & 0,7 & $0,2-1,2$ & 0,7 & 1,0 \\
\hline & $\mathrm{C}$ & $0,5-0,8$ & 0,7 & 0,8 & $0,6-1,0$ & 0,9 & 1,2 \\
\hline Candida não & A & $0,3-0,9$ & 0,7 & 0,9 & $0,3-1,5$ & 1,3 & 1,5 \\
\hline \multirow[t]{2}{*}{ albicans } & B & $0,2-1,2$ & 0,7 & 1,0 & $0,3-1,5$ & 0,8 & 1,4 \\
\hline & $\mathrm{C}$ & $0,2-0,7$ & 0,5 & 0,7 & $0,3-1,3$ & 0,7 & 1,1 \\
\hline \multirow[t]{3}{*}{ Total } & $\bar{A}$ & $0,3-0,9$ & 0,7 & 0,9 & $0,3-1,5$ & 1,3 & 1,5 \\
\hline & B & $0,2-1,2$ & 0,7 & 1,0 & $0,2-1,5$ & 0,9 & 1,4 \\
\hline & C & $0,2-0,8$ & 0,7 & 0,8 & $0,3-1,3$ & 0,7 & 1,2 \\
\hline \multicolumn{8}{|c|}{ Candida parapsilosis } \\
\hline ATCC 22019 & - & $0,6-0,7$ & ND & ND & $0,6-0,8$ & ND & ND \\
\hline \multicolumn{8}{|l|}{ Candida krusei } \\
\hline ATCC 6258 & - & 0,9 & ND & ND & 1,2 & ND & ND \\
\hline
\end{tabular}

AM3: (antibiotic medium 3), CIM: concentração inibitória mínima, CFM: concentração fungicida mínima, ND: não determinado, A (Hospital Universitário de Santa Maria), B (Hospital de Clínicas de Porto Alegre), C (Santa Casa, Complexo Hospitalar de Porto Alegre).
Tabela 3 - Avaliação da suscetibilidade de Candida spp, procedentes de três hospitais do Rio Grande do Sul, frente à anfotericjna $\mathrm{B}$, utilizandose o Caldo YNBd.

\begin{tabular}{|c|c|c|c|c|c|c|c|}
\hline \multirow[b]{2}{*}{ Espécies } & \multirow[b]{2}{*}{ Hospitais } & \multicolumn{3}{|c|}{$\operatorname{CIM}(\mu \mathrm{g} / \mathrm{ml})$} & \multicolumn{3}{|c|}{$\operatorname{CFM}(\mu \mathrm{g} / \mathrm{ml})$} \\
\hline & & variação & CIM50 & CIM90 & variação & CIM50 & CIM90 \\
\hline$\overline{\text { Candida }}$ & $\bar{A}$ & $0,4-0,1$ & 0,5 & 0,8 & $0,5-1,2$ & 0,7 & 1,0 \\
\hline \multirow[t]{2}{*}{ albicans } & B & $0,2-1,0$ & 0,7 & 0,9 & $0,2-1,2$ & 0,8 & 1,1 \\
\hline & $\mathrm{C}$ & $0,5-0,9$ & 0,7 & 0,9 & $0,6-1,0$ & 0,8 & 1,0 \\
\hline Candida não & A & $0,3-0,9$ & 0,6 & 0,8 & $0,6-1,2$ & 0,7 & 1,2 \\
\hline \multirow[t]{3}{*}{ albicans } & B & $0,2-1,0$ & 0,7 & 0,9 & $0,2-1,2$ & 0,9 & 1,2 \\
\hline & $\mathrm{C}$ & $0,3-0,8$ & 0,6 & 0,8 & $0,3-1,1$ & 0,7 & 1,0 \\
\hline & A & $0,3-1,0$ & 0,6 & 0,8 & $0,5-1,2$ & 0,7 & 1,2 \\
\hline \multirow[t]{2}{*}{ Total } & B & $0,2-1,0$ & 0,7 & 0,9 & $0,2-1,2$ & 0,9 & 1,2 \\
\hline & C & $0,3-0,9$ & 0,7 & 0,9 & $0,3-1,1$ & 0,8 & 1,0 \\
\hline \multicolumn{8}{|c|}{ Candida parapsilosis } \\
\hline ATCC 22019 & - & 0,6 & ND & ND & 0,9 & ND & ND \\
\hline \multicolumn{8}{|c|}{ Candida kruse $i$} \\
\hline ATCC 6258 & - & 1,0 & ND & ND & 1,4 & ND & ND \\
\hline
\end{tabular}

YNBd: (Yeast Nitrogen Base dextrose), CIM: concentração inibitória mínima, CFM: concentração fungicida mínima, ND: não determinado. A (Hospital Universitário de Santa Maria), B (Hospital de Clínicas de Porto Alegre), C (Santa Casa, Complexo Hospitalar de Porto Alegre).

das CFMs menor (10 concentrações) do que a obtida com o AM3 (13 concentrações). A vantagem de se obter ampla distribuição de CIMs ou CFMs está na detecção de isolados resistentes. Com este fim, as CIMs obtidas no caldo AM3 reclassificaram como resistentes a anfotericina $\mathrm{B}$, um isolado de Candida tropicalis $(\mathrm{CIM}=1,1 \mu \mathrm{g} / \mathrm{ml} ; \mathrm{CFM}=1,5 \mu \mathrm{g} / \mathrm{ml})$ e outro de Candida guilliermondii $(\mathrm{CIM}=1,2 \mu \mathrm{g} / \mathrm{ml}$; CFM= $1,4 \mu \mathrm{g} / \mathrm{ml})$; as CFMs também foram elevadas, embora, outros isolados com CIMs dentro da faixa sensível requeressem CFMs similares a das resistentes.

Excetuando-se Candida albicans, o número de isolados representando as demais espécies, era muito heterogêneo; assim, para fins de comparações estatísticas, tais espécies foram reunidas no grupo Candida não albicans. Ao se comparar o perfil de suscetibilidade de Candida albicans com o grupo Candida não albicans, o teste de Wilcoxon não indicou diferenças significativas com nenhum dos três meios de cultura empregados. Cabe ressaltar, ainda, que no grupo não albicans, a espécie Candida parapsilosis evidenciou nítida sensibilidade à anfotericina $\mathrm{B}$, nos 3 meios empregados.

Finalmente, comparou-se as variações de suscetibilidade a anfotericina B de acordo com a instituição hospitalar, através dos 3 meios de cultura. A Tabela 4 dispõe estas comparações após análise estatística pelo teste de Kruskal-Wallis. Estes resultados indicam que existem diferenças nos perfis de suscetibilidade de Candida procedentes de diferentes hospitais. De modo geral, os isolados do Hospital Universitário de Santa Maria foram significativamente menos sensíveis a anfotericina $B$ do que os isolados da Santa Casa-Complexo Hospitalar de Porto Alegre; em três parâmetros, os isolados da Santa Casa-Complexo Hospitalar de Porto Alegre foram menos sensíveis do que os do Hospital de Clínicas de Porto Alegre. Em nenhuma comparação os isolados do Hospital de Clínicas de Porto Alegre foram os menos sensíveis a anfotericina B quando comparados aos demais isolados. 
Tabela 4 - Comparação dos parâmetros de suscetibilidade (CIMs e CFMs) de Candida spp, procedentes de três bospitais do Rio Grande do Sul, frente a anfotericina B, em função dos meios utilizados nos testes.

\begin{tabular}{|c|c|c|c|c|c|c|}
\hline \multirow{2}{*}{$\begin{array}{l}\text { Grupos de Candida spp } \\
\text { versus hospitais }\end{array}$} & \multicolumn{2}{|c|}{ RPMI 1640} & \multicolumn{2}{|c|}{ AM3 } & \multicolumn{2}{|c|}{ YNBd } \\
\hline & CIM & CFM & CIM & CFM & CIM & CFM \\
\hline \multicolumn{7}{|l|}{$\overline{\text { Candida albicans }}$} \\
\hline $\mathrm{A} \times \mathrm{B} \times \mathrm{C}$ & NS & NS & $C>B$ & $\mathrm{C}>\mathrm{B}$ & NS & $\mathrm{C}>\mathrm{B}$ \\
\hline \multicolumn{7}{|l|}{ Candida não albicans } \\
\hline $\mathrm{A} \times \mathrm{B} \times \mathrm{C}$ & $\mathrm{A}>\mathrm{C}$ & $\mathrm{A}>\mathrm{C}$ & $A>C$ & $\mathrm{~A}>\mathrm{C}$ & NS & NS \\
\hline Gênero Candida & $A>C$ & $\mathrm{~A}>\mathrm{C}$ & $A>B$ & $A>B$ & NS & $\mathrm{NS}$ \\
\hline
\end{tabular}

Teste de Kruskal-Wallis $(\mathrm{p}<0,05)$, CIM: concentração inibitória mínima, CFM: concentração fungicida mínima, NS: diferenças observadas não foram significantes, A (Hospital Universitário de Santa Maria), B (Hospital de Clínicas de Porto Alegre), C (Santa Casa, Complexo Hospitalar de Porto Alegre).

A Tabela 4 evidencia, ainda, que o caldo AM3 permitiu detectar mais diferenças entre os isolados do que o Caldo RPMI 1640. 0 caldo YNBd foi o menos eficiente na detecção das diferenças de suscetibilidade entre os isolados de Candida spp.

\section{DISCUSSÃo}

0 fenômeno da resistência aos antifúngicos tem sido bastante estudado nos últimos anos, devido a recente padronização dos testes de suscetibilidade para fungos leveduriformes ${ }^{17}$ e fungos filamentosos ${ }^{18}$.

Casos de insucesso terapêutico com anfotericina B têm sido relatados bem como casos de espécies de Candida resistentes foram publicados entre 1968 e $1985^{278}$. Em que pese a importância e inusitado destes achados, tais relatos foram baseados em testes executados com técnicas não padronizadas. A partir de 1997, com a disponibilidade de uma técnica padronizada, os testes de Candida spp a anfotericina B têm gerado interpretações conflitantes porque estas metodologias não definiram pontos de corte (breakpoints) que permitam classificar os isolados como sensíveis ou resistentes à anfotericina $\mathrm{B}^{1011} 1522$. A principal justificativa para a não definição de breakpoints é que o caldo RPMI 1640, indicado no documento M27-A2 ${ }^{17}$ tem sido apontado como um obstáculo na detecção de isolados resistentes a anfotericina $\mathrm{B}$. Todavia, falhas às terapêuticas com anfotericina $B$ continuam a ser relatadas, com isolados evidenciando, majoritariamente, sensibilidade in vitro a este poliênico ${ }^{11141928}$. O caldo RPMI 1640, gera uma faixa de CIMs muito estreita ou compacta, impossibilitando as definições para breakpoints ${ }^{11222728}$. Por outro lado, tem sido sugerido que CIMs > 1 $\mu \mathrm{g} / \mathrm{ml}$ se relacionam com resistência ${ }^{21} 2728$ 29; a inadequação do caldo RPMI 1640 determinou que o CLSI no documento M27-A2 já sugerisse a avaliação da suscetibilidade com 0 antibiotic medium 3 (AM3) ${ }^{11} 1728$. As dificuldades na detecção da resistência à anfotericina B pela M27-A2 tem estimulado a utilização de outras metodologias: o $E$ teste tem sido o método mais sensível e prático para a detecção desta resistência ${ }^{41130}$. Estudos com curvas de morte e CFMs também têm sido avaliados com este propósito, evidenciando resultados animadores $^{1926}$.
No presente estudo, propusemos comparar o desempenho do Caldo RPMI 1640 com o AM3 e o caldo YNBd, ao se avaliar a suscetibilidade de Candida spp à anfotericina B. Basicamente, seguiu-se a metodologia do documento M27-A2. Entretanto, o número das concentrações de anfotericina $\mathrm{B}$ foi aumentado, incluindo-se: 0,1-0,2-0,3-0,4-0,5-0,6-0,7-0,8-0,9-1,0-1,1-1,2-

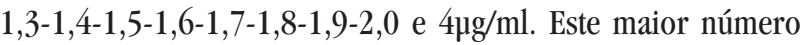
de concentrações avaliadas resultou em amplas faixas de CIMs e CFMs, embora, o caldo RPMI 1640 tenha gerado resultados mais agrupados do que o AM3, confirmando-se prévias constatações ${ }^{1628}$. Amplas faixas de CIMs e/ou de CFMs são vantajosas para definição de pontos de corte. 0 antibiotic medium 3 foi o meio que permitiu melhor diferenciação da susceptibilidade entre os grupos de Candida. A capacidade deste meio em descompactar a faixa de CIMs geradas pelo caldo RPMI 1640 tem sido constatada, todavia o fato de se constituir em meio complexo, sem composição química completamente conhecida, tem gerado problemas de reprodutibilidade de resultados quando as mesmas amostras são testadas com lotes diferentes do $\mathrm{AM} 3^{17}$.

O maior número de concentrações de anfotericina incluídas nos ensaios, determinou a obtenção de amplas faixas de suscetibilidade com todos os três meios avaliados; estudos de correlação entre testes de suscetibilidade a anfotericina B e desfecho das candidemias poderão ser mais proveitosos com a inclusão de maior número de concentrações de anfotericina B nos ensaios, pois, resultam em maiores possibilidades para definição de pontos de corte e/ou detecção do fenômeno da resistência.

A comparação do perfil de suscetibilidade de todos os isolados de Candida albicans com o grupo nomeado Candida não albicans não indicou diferenças significativas, com qualquer dos meios empregados. Este resultado vem expressar a heterogeneidade do grupo não albicans; entre elas, Candida glabrata, naturalmente menos sensível ao fluconazol e anfotericina B, e Candida krusei, a qual é considerada multirresistente, pois, além de resistente ao fluconazol, somente $8 \%$ dos isolados evidenciam a anfotericina $\mathrm{B}$ CIM $\leq 1 \mu \mathrm{g} / \mathrm{ml}^{24}{ }^{30}$. A resistência observada em Candida guilliermondii $(\mathrm{CIM}=1,2)^{30}$ constituiu-se num achado inusitado, pois, a resistência desta espécie a anfotericina B costuma ser pouco expressiva; num estudo com 102 isolados, Tietz e cols ${ }^{30}$ detectaram pelo E test apenas 2 isolados resistentes.

Estudos de suscetibilidade de Candida spp a antifúngicos, incluindo anfotericina B, têm sido realizados no Brasil e no Rio Grande do Sul, sem, todavia, detecção de resistência a este poliênico. No Rio Grande do Sul, Antunes e cols ${ }^{1}$ avaliaram a suscetibilidade de 120 isolados de candidemias utilizando procedimentos padronizados pelo CLSI (M27-A2) ${ }^{17}$. Todos os isolados evidenciaram à anfotericina $\mathrm{B}$ CIMs $\leq 1 \mu \mathrm{g} / \mathrm{mL}$, considerados sensíveis. Recentemente, Colombo e cols ${ }^{6}$ num estudo multicêntrico sobre candidemias, envolvendo 11 centros médicos do Brasil, avaliaram 712 isolados de candidemia através da técnica M27-A2 ${ }^{17}$. Frente à anfotericina $\mathrm{B}$, não consideraram nenhum achado de resistência, embora 5 isolados de Candida pelliculosa tenham extendido a faixa de suscetibilidade de $0,125 \mu \mathrm{g} / \mathrm{mL}$ a $2 \mu \mathrm{g} / \mathrm{mL}$.

Nos Estados Unidos, Ostrosky-Zeichner e cols ${ }^{20}$ num estudo multicêntrico sobre candidemias, empregaram o caldo AM3 
para avaliação da suscetibilidade à anfotericina B; entre 2000 isolados detectaram $0,8 \%$ de resistência envolvendo as espécies Candida glabrata, Candida tropicalis, Candida parapsilosis e Candida krusei.

Ao compararmos a suscetibilidade à anfotericina $\mathrm{B}$ de isolados de Candida procedentes de três hospitais do Rio Grande do Sul, constatou-se que haviam diferenças significativas entre estas amostras. Embora os dois isolados considerados resistentes a anfotericina B (Candida tropicalis e Candida guilliermondii) fossem oriundas do Hospital de Clínicas de Porto Alegre (Hospital B), os isolados desta instituição não se destacaram como os menos sensíveis. Os isolados do Hospital Universitário de Santa Maria (Hospital A) foram significativamente menos sensíveis do que os procedentes da Santa Casa - Complexo Hospitalar de Porto Alegre (Hospital C) em oito comparações, apesar de não terem sido detectados isolados resistentes.

De modo geral, os parâmetros CIM e CFM à anfotericina B permitiram demonstrar as mesmas variações de suscetibilidade de Candida spp à anfotericina B entre os hospitais estudados (Tabela 4). As causas das variações de suscetibilidade são desconhecidas; por outro lado, apontam para a necessidade de vigilância da suscetibilidade a anfotericina $\mathrm{B}^{23}$ utilizando-se, também, novas propostas que garantam melhorar a avaliação da emergência do fenômeno da resistência a este poliênico $0^{416192628}$.

\section{REFERÊNCIAS}

1. Antunes AGV, Pasqualotto AC, Díaz MC, d' Azevedo PA, Severo LC. Candidemia in a Brazilian tertiary care hospital: species distribution and antifungal susceptibility patterns. Revista do Instituto de Medicina Tropical de São Paulo 46: 239-241, 2004.

2. Bodenhoff J. Development of strains of genus Candida and genus Torulopsis resistant to antimycotics. Acta Pathologica Microbiologica Scandinavica 75: 622-630, 1969

3. Canuto MM, Rodero FG. Antifungal drug resitance to azoles and polyenes. The Lancet Infectious Diseases 2: 550-563, 2002.

4. Clancy CJ, Nguyen MH. Correlation between in vitro susceptibility determined by E test and response to therapy with amphotericin B: results from a multicenter prospective study of candidemia. Antimicrobial Agents Chemotherapy 43: 12891290, 1999 .

5. Collin B, Clancy CJ, Nguyen MH. Antifungal resistance in non-albicans Candida species. Drug Resistance Updates 2: 9-14, 1999.

6. Colombo AL, Nucci M, Park BJ, Nouér AS, Arthington-Skaggs B, Matta D, Warnock D, Morgan J. Epidemiology of candidemia in Brazil: a nationwide sentinel surveillance of candidemia in eleven medical centers. Journal of Clinical Microbiology 44: 2816-2823, 2006.

7. Dick JD, Merz WG, Saral R. Incidence of polyene resistant yeasts recovered from clinical specimens. Antimicrobial Agents Chemotherapy 18: 158-163, 1980.

8. Dick JD, Rosengard BR, Merz WG, Stuart RK, Hutchins GM, Saral R. Fatal disseminated candidiasis to amphotericin B resistant $C$. guilliermondii. Annals of Internal Medicine 102: 67-68, 1985.

9. Dodds Ashley ES, Lewis R, Lewis JS, Martin C, Andes D. Pharmacology of systemic antifungal agents. Clinical Infectious Diseases 43 (suppl 1): S28-S39, 2006

10. Ellis D. Amphotericin B: spectrum and resistance. Journal of Antimicrobial Chemotherapy 49 (suppl 1): S7-S10, 2002.

11. Hospenthal DR, Murray CK, Rinaldi MG. The role of antifungal susceptibility testing in the therapy of candidiasis. Diagnostic Microbiology and Infectious Diseases 48: 1153-1160, 2004.
12. Krogh-Madsen M, Arendrup MC, Heslet L, Knudsen JD. Amphotericin B and caspofungin resistance in Candida glabrata isolates recovered from a critically ill patient. Clinical Infectious Diseases 42: 938-944, 2006.

13. Law D, Moore CB, Denning DW. Amphotericin B resistance testing Candida spp a comparison of methods. Journal of Antimicrobial Chemotherapy 40: 109-112, 1997.

14. Liu KH, Wu CJ, Chou CH, Lee HC, Lee NY, Hung ST, Ko WC. Refractory candidal meningitis in an immunocompromised patient cured by caspofungin. Journal of Clinical Microbiology 42: 5950-5953, 2004.

15. Loeffler J, Stevens DA. Antifungal drug resistance. Clinical Infectious Diseases 30 (suppl 1): S31-41, 2003.

16. Lozano-Chiu M, Paetznick VL, Ghannoum MA, Rex JH. Detection of resistance to amphotericin B among Cryptococcus neoformans clinical isolates: performances of three different media assessed by using $\mathrm{E}$ test and National Committee for Clinical Laboratory Standards M27-A methodologies. Journal of Clinical Microbiology 36: 2817-2822, 1998.

17. National Committee for Clinical Laboratory Standards. Reference method for broth dilution antifungal susceptibility testing of yeasts. Approved standard M27-A2. National Committee for Clinical Laboratory Standards, Wayne, PA. 2002.

18. National Committee for Clinical Laboratory Standards. Reference method for broth dilution antifungal susceptibility testing of filamentous fungi. Approved standard M38-A. National Committee for Clinical Laboratory Standards, Wayne, PA. 2002.

19. Nguyen MH, Clancy CJ, Yu VL, Yu YV, Morris AJ, Snydman DR, Sutton DA, Rinaldi MG. Do in vitro susceptibility data predict the microbiologic response 0 amphotericin B? Results of a prospective study of patients with Candida fungemia. Journal of Infectious Diseases 177: 425-430, 1998

20. Ostrosky-Zeichner L, Rex JH, Hamil PG, Larsen RA, Horowitz HW, Powderly WG, Hyslop N, Kauffman CA, Clerly J, Mangino JE, Lee J. Antifungal susceptibility of 2,000 bloodstream Candida isolates in the United States. Antimicrobial Agentes Chemotherapy 47: 3149-3154, 2003.

21. Pappas PG, Rex JH, Sobel JD, Filler SG, Dismukes WE, Walsh TJ, Edwards JE. Guidelines for treatment of candidiasis. Clinical Infectious Diseases 38: 161-189, 2004.

22. Park BJ, Arthington-Skaggs BA, Hajjeh RA, Iqbal N, Ciblak MA, Lee-Young W, Hariston MD, Phelan M, Plikaytis BD, Sofair AN, Harrison LH, Fridkin SK, Warnock DW. Evaluation of amphotericin B interpretative breakpoints for Candida bloodstream isolates by correlation with therapeutic outcome. Antimicrobial Agents Chemotherapy 50: 1287-1292, 2006.

23. Pfaller MA, Diekema DJ. Role of sentinel surveillance of candidemia: trends in species distribution and antifungal susceptibility. Journal of Clinical Microbiology 40: 3551-3557, 2002

24. Pfaller MA, Diekema DJ. Rare and emerging opportunistic fungal pathogens: concern for resistance beyond Candida albicans and Aspergillus fumigatus. Journal of Clinical Microbiology 42: 4419-4431, 2004.

25. Pfaller MA, Diekema DJ. Epidemiology of invasive candidiasis: persistent public health problem. Clinical Microbiology Reviews 20: 133-163, 2007.

26. Pfaller MA, Sheehan DJ, Rex JH. Determination of fungicidal activities against yeasts and molds: lessons learned from bactericidal testing and need for standardization. Clinical Reviews Microbiology 17: 268-280, 2004.

27. Rex JH, Cooper CR, Merz WG, Galgiani JN, Anaissie EJ. Detection of Amphotericin B-resistant Candida isolates in a broth-based system. Antimicrobial Agents Chemotherapy 39: 906-909, 1995.

28. Rex JH, Pfaller MA, Walsh TJ, Chatuverdi V, Espinel-Ingroff A, Ghannoum MA, Gosey LL, Odds FC, Rinaldi MG, Sheehan DJ, Warnock DW. Antifungal susceptibility testing: practical aspects and current challenges. Clinical Microbiology Reviews 14: 643-658, 2001.

29. Spelberg BJ, Filler SG, Edwards Jr JE. Current treatment strategies for disseminated candidiasis. Clinical Infectious Diseases 2: 244-251, 2006

30. Tietz HJ, Czaida V, Sterry W. Case report. Osteomyelitis caused by highly-resistant Candida guilliermondii. Mycoses 42: 577-580, 1999.

31. Wey SB, Mori M, Pfaller MA, Woolson RF, Wenzel RP. Hospital-acquired candidemia. The attributable mortality and excess length of stay. Archives of Internal Medicine 148: 2642-2645, 1998 